\title{
Ranging fusion for accurating state of the art robot localization
}

\author{
Hamed Bastani ${ }^{1}$ and Hamid Mirmohammad-Sadeghi ${ }^{2}$ \\ 1 Jacobs University Bremen, Germany \\ 2Isfahann University of Technology, Iran
}

\section{Introduction}

Generally speaking, positioning and localization give somehow the same comprehension in terminology. They can be defined as a mechanism for realizing the spatial relationship between desired features. Independent from the mechanisms themselves, they all have certain requirements to fulfil. Scale of measurements and granularity is one important aspect to be investigated. There are limitations, and on the other hand expectations, depending on each particular application. Accuracy gives the closeness of the estimated solution with respect to the associated real position of a feature in the work space (a.k.a ground truth position). Consistency of the realized solution and the ground truth, is represented by precision. Other parameters are still existing which leave more space for investigation depending on the technique used for localization, parameters such as refresh rate, cost (power consumption, computation, price, infrastructure installation burden, ...), mobility and adaptively to the environment (indoor, outdoor, space robotics, underwater vehicles, ...) and so on.

From the mobile robotics perspective, localization and mapping are deeply correlated. There is the whole field of Simultaneous Localization and Mapping (SLAM), which deals with the employment of local robot sensors to generate good position estimates and maps; see (Thrun, 2002) for an overview. SLAM is also intensively studied from the multi robot perspective. This is while SLAM requires high end obstacle detection sensors such as laser range finders and it is computationally quite expensive.

Aside from SLAM, there are state of the art positioning techniques which can be anyhow fused in order to provide higher accuracy, faster speed and to be capable of dealing with systems with higher degrees of complexity. Here, the aim is to first of all survey an introductory overview on the common robot localization techniques, and particularly then focus on those which employ a rather different approach, i.e ranging by means of specially radio wireless technology. It will be shortly seen that mathematical tools and geometrical representation of a graph model containing multiple robots as the network nodes, can be considered a feature providing higher positioning accuracy compared to the traditional methods. Generally speaking, such improvements are scalable and also provide robustness against variant inevitable disturbing environmental features and measurement noise. 


\section{State of the Art Robot Localization}

Localization in the field of mobile robotics is vast enough to fall into unique judgements and indeed categorization. There are plenty of approaches tackling the problem from different perspectives. In order to provide a concrete understanding of grouping, we start facing the localization mechanisms with a form of categorization as follows.

- Passive Localization: where already present signals are observed and processed by the system in order to deduce location of desired features. Clearly, depending on the signal's specifications, special sensory system as well as available computation power, certain flexibility is required due to passiveness.

- Active Localization: in which, the localization mechanism itself generates and uses its own signals for the localization purpose.

Preference is up to the particular application where it may choose a solution which falls in either of the above classes. However, a surface comparison says the second approach would be more environmental independent and therefore, more reliable for a wider variety of applications. This again will be a tradeoff for some overhead requirements such as processing power, computation resources and possibly extra auxiliary hardware subunits. From another side of assessment, being utilized hardware point of view, we can introduce the techniques below, which each itself is either passive or active:

1. Dead reckoning: uses encoders, principally to realize translational movements from rotational measurements based on integration. Depending on the application, there are different resolutions defined. This class is the most basic but at the same time the most common one, applied in mobile robotics. Due to its inherit characteristics, this method is considered noisy and less robust. On the other hand, due to its popularity, there has been enough research investment to bring about sufficient improvements for the execution and results quality of this technique (e.g. (Lee) and (Heller) can be referred to).

2. INS methods: which are based on inertial sensors, accelerometers and detectors for electromagnetic field and gravity. They are also based on integration on movement elements, therefore, may eventually lead to error accumulation especially if drift prune sensors are used. Due to vast field of applications, these methods also enjoyed quite enough completeness, thanks to the developed powerful mathematical tools. (Roos, 2002) is for example offering one of these complementary enhancements.

3. Vision and visual odometery: utilizing a single camera, stereo vision or even omni directional imaging, this solution can potentially be useful in giving more information than only working as the localization routine. This solution can considerably become more computationally expensive, especially if employed in a dynamic environment or expected to deal with relatively-non-stationary features. It is however, considered a popular and effective research theme lately, and is enhanced significantly by getting backup support from signal processing techniques, genetic algorithms, as well as evolutionary and learning algorithms. 
4. Ranging: employing a distance measurement media that can be either laser, infrared, acoustic or radio signals. Ranging can be done using different techniques; recording signal's Time of Flight, Time Difference of Arrival or Round Trip Time of Flight of the beamed signal, as well as its Angle of Arrival. This class is under main interest to be fused properly for increasing efficiency and accuracy of the traditional methods.

There are some less common approaches which indirectly can still be categorized in the classes above. Itemized reference to them is as the following.

Doppler sensors can measure velocity of the moving objects. Principally speaking, a sinusoidal signal is emitted from a moving platform and the echo is sensed at a receiver. These sensors can use ultra sound signal as carrier or radio waves, either. Related to the wavelength, resolution and accuracy may differ; a more resolution is achieved if smaller wavelength (in other words higher frequency) is operational. Here again, this technique works based on integrating velocity vectors over time.

Electromagnetic trackers can determine objects' locations and orientations with a high accuracy and resolution (typically around $1 \mathrm{~mm}$ in coordinates and $0.2 \circ$ in orientation). Not only they are expensive methods, but also electromagnetic trackers have a short range (a few meters) and are very sensitive to presence of metallic objects. These limitations only make them proper for some fancy applications such as body tracking computer games, animation studios and so on.

Optical trackers are very robust and typically can achieve high levels of accuracy and resolution. However, they are most useful in well-constrained environments, and tend to be expensive and mechanically complex. Example of this class of positioning devices are head tracker system (Wang et. al, 1990).

Proper fusion of any of the introduced techniques above, can give higher precision in localization but at the same time makes the positioning routine computationally more expensive. For example (Choi \& Oh, 2005) combines sonar and vision, (Carpin \& Birk, 2004) fuses odometery, INS and ranging, (Fox et. al, 2001) mixes dead reckoning with vision, and there can be found plenty of other practical cases. Besides, each particular method can not be generalized for all applications and might fail under some special circumstances. For instance, using vision or laser rangefinders, should be planned based on having a rough perception about the working environment beforehand (specially if system is working in a dynamic work space. If placed out and not moving, the strategy will differ, eg. in (Bastani et. al, 2005)). Solutions like SLAM which overcome the last weakness, need to detect some reliable features from the work space in order to build the positioning structure (i.e. an evolving representation called as the map) based on them. This category has a high complexity and price of calculation. In this vain, recent technique such as pose-graph put significant effort on improvements (Pfingsthhorn \& Birk, 2008). Concerning again about the environmental perception; INS solutions and accelerometers, may fail if the work space is electromagnetically noisy. Integrating acceleration and using gravity specifications in addition to the dynamics of the system, will cause a potentially large accumulative error in positioning within a long term use, if applied alone. 


\section{Ranging Technologies and Wireless Media}

The aim here is to refer to ranging techniques based on wireless technology in order to provide some network modelling and indeed realization of the positions of each node in the network. These nodes being mobile robots, form a dynamic (or in short time windows static) topology.

Different network topologies require different positioning system solutions. Their differences come from physical layer specifications, their media access control layer characteristics, some capabilities that their particular network infrastructure provides, or on the other hand, limitations that are imposed by the network structure. We first very roughly categorizes an overview of the positioning solutions including variety of the global positioning systems, those applied on cellular and GSM networks, wireless LANs, and eventually ad hoc sensor networks.

\subsection{Wireless Media}

Two communicating nodes compose the simplest network where there is only one established link available. Network size can grow by increasing the number of nodes. Based on the completeness of the topology, each node may participate in establishing various number of links. This property is used to define degree of a node in the network. Link properties are relatively dependent on the media providing the communication. Bandwidth, signal to noise ratio (SNR), environmental propagation model, transmission power, are some of such various properties. Figure 1 summarizes most commonly available wireless communication media which currently are utilized for network positioning, commercially or in the research fields. With respect to the platform, each solution may provide global or local coordinates which are eventually bidirectionally transformable. Various wireless platforms - based on their inherent specifications and capabilities, may be used such that they fit the environmental conditions and satisfy the localization requirements concerning their accessibility, reliability, maximum achievable resolution and the desired accuracy.

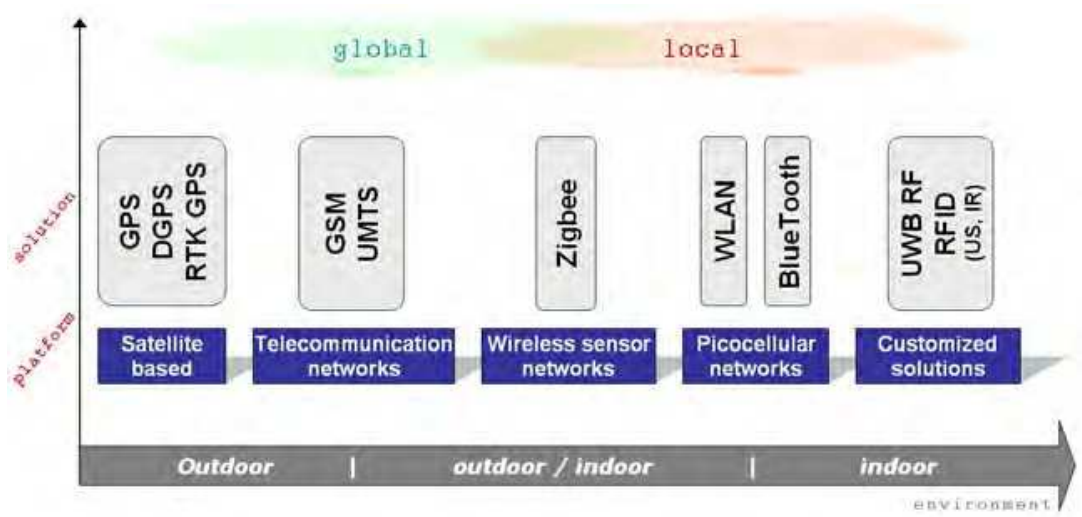

Fig. 1. Sweeping the environment from outdoor to indoor, this figure shows how different wireless solutions use their respective platforms in order to provide positioning. They all indeed use some ranging technique for the positioning purpose, no matter time of flight or received signal strength. Depending on the physical size of the cluster, they provide local or global positions. Anyhow these two are bidirectional transformable. 
Obviously, effectiveness and efficiency of a large scale outdoor positioning system is rather different than a small scale isolated indoor one. What basically differs is the environment which they may fit better for, as well as accuracy requirements which they afford to fulfil. On the $\mathrm{x}$-axis of the diagram in figure 1, moving from outdoor towards indoor environment, introduced platforms become less suitable specially due to the attenuation that indoor environmental conditions apply to the signal. This is while from right to left of the $x$-axis in the same diagram, platforms and solutions have the potential to be customized for outdoor area as well. The only concern is to cover a large enough area outdoor, by pre-installing the infrastructure.

The challenge is dealing with accurate indoor positioning where maximum attenuation is distorting the signal and most of the daily, surveillance and robotics applications are utilized. In this vein, we refer to the WLAN class and then for providing enhancement and more competitive accuracy, will turn to wireless sensor networks.

\subsection{Wireless Local Area Networks}

Very low price and their common accessibility have motivated development of wireless LAN-based indoor positioning systems such as Bluetooth and Wi-Fi. (Salazar, 2004) comprehensively compares typical WLAN systems in terms of markets, architectures, usage, mobility, capacities, and industrial concerns. WLAN-based indoor positioning solutions mostly depend on signal strength utilization. Anyhow they have either a client-based or client-assisted design.

Client-based system design: Location estimation is usually performed by RF signal strength characteristics, which works much like pattern matching in cellular location systems. Because signal strength measurement is part of the normal operating mode of wireless equipment, as in Wi-Fi systems, no other hardware infrastructure is required. A basic design utilizes two phases. First, in the offline phase, the system is calibrated and a model is constructed based on received signal strength (RSS) at a finite number of locations within the targeted area. Second, during an online operation in the target area, mobile units report the signal strengths received from each access point (AP) and the system determines the best match between online observations and the offline model. The best matching point is then reported as the estimated position.

Client-assisted system design: To ease burden of system management (provisioning, security, deployment, and maintenance), many enterprises prefer client-assisted and infrastructurebased deployments in which simple sniffers monitor client's activity and measure the signal strength of transmissions received from them (Krishnan, 2004). In client-assisted location system design, client terminals, access points, and sniffers, collaborate to locate a client in the WLAN. Sniffers operate in passive scanning mode and sense transmissions on all channels or on predetermined ones. They listen to communication from mobile terminals and record time-stamped information. The sniffers then put together estimations based on a priory model. A client's received signal strength at each sniffer is compared to this model using nearest neighbour searching to estimate the clients location (Ganu, 2004). In terms of system deployment, sniffers can either be co-located with APs, or, be located at other 
positions and function just like the Location Management Unit in a cellular-based location system.

\subsection{Ad hoc Wireless Sensor Networks}

Sensor networks vary significantly from traditional cellular networks or similars. Here, nodes are assumed to be small, inexpensive, homogeneous, cooperative, and autonomous. Autonomous nodes in a wireless sensor network (WSN) are equipped with sensing (optional), computation, and communication capabilities. The key idea is to construct an ad hoc network using such wireless nodes whereas nodes' locations can be realized. Even in a pure networking perspective, location-tagged information can be extremely useful for achieving some certain optimization purposes. For example (Kritzler, 2006) can be referred to which proposes a number of location-aware protocols for ad hoc routing and networking. It is especially difficult to estimate nodes' positions in ad hoc networks without a common clock as well as in absolutely unsynchronized networks. Most of the localization methods in the sensor networks are based on RF signals' properties. However, there are other approaches utilizing Ultra Sound or Infra Red light instead. These last two, have certain disadvantages. They are not omnidirectional in broadcasting and their reception, and occlusion if does not completely block the communication, at least distorts the signal significantly. Due to price flexibilities, US methods are still popular for research applications while providing a high accuracy for in virtu small scale models.

Not completely inclusive in the same category however there are partially similar techniques which use RFID tags and readers, as well as those WSNs that work based on RF UWB communication, all have proven higher potentials for indoor positioning. An UWB signal is a series of very short baseband pulses with time durations of only a few nanoseconds that exist on all frequencies simultaneously, resembling a blast of electrical noise (Fontanaand, 2002). The fine time resolution of UWB signals makes them promising for use in high-resolution ranging. In this category, time of flight is considered rather than the received signal strength. It provides much less unambiguity but in contrast can be distorted by multipath fading. A generalized maximum-likelihood detector for multipaths in UWB propagation measurement is described in (Lee, 2002). What all these techniques are suffering from is needing a centralized processing scheme as well as a highly accurate and synchronized common clock base. Some approaches are however tackling the problem and do not concern time variant functions. Instead, using for example RFID tags and short-range readers, enables them to provide some proximity information and gives a rough position of the tag within a block accuracy/resolution (e.g. the work by (Fontanaand, 2002) with very short range readers for a laboratory environment localization). The key feature which has to be still highlighted in this category is the overall cost of implementation.

\section{Infra Structure Principles}

In the field of positioning by means of radio signals, there are various measurement techniques that are used to determine position of a node. In a short re-notation they are divided into three groups:

- Distance Measurements: ToF, TDoA, RSS

- Angle Measurements: AoA

- Fingerprinting: RSS patterns (radio maps) 
Distance and angle measurement methods are the mostly used metrics for outdoor location systems. Distance measurements use the path loss model and ToF measurements to determine a location. Angle Measurements are based on knowing the angle of incidence of the received signal. However, this class requires directional antennas and antenna arrays to measure the angle of incidence. That makes this option not very viable for a node with highmobility. For smaller scale applications, this method can be utilized by means of ESPAR (Electronically Steerable Parasitic Array Radiator) antennas. Such an antenna steers autonomously its beam toward the arrival direction of desired radio waves and steers the nulls of the beam toward the undesired interfering waves (Ging, 2005). The versatile beam forming capabilities of the ESPAR antenna allows to reduce multipath fading and makes accurate reading for direction of arrival. There are not too much of applicable experiences for indoor mobile robotics, (Shimizu, 2005) for example, applies it on search and rescue robotics for urban indoor environment.

Distance and Angle measurements work only with direct line-of-sight signals from the transmitter to the receiver, indeed being widely practical for outdoor environments. For indoors, channel consists of multipath components, and the mobile station is probably surrounded by scattering objects. For these techniques to work, a mobile node has to see at least three signal sources, necessarily required for triangulation.

Distance measurement techniques in the simplest case, will end up to multilateration in order to locate position of a desired point, no matter being a transmitter or a receiver. Collaborative multilateration (also referred to as N-hop multilateration) consists of a set of mechanisms that enables nodes to find several hops away from beacon nodes to collaborate with each other and potentially estimate their locations within desired accuracy limits. Collaborative multilateration is presented in two edges of computation models, centralized and distributed. These can be used in a wide variety of network setups from fully centralized where all the computation takes place at a base station, locally centralized (i.e., computation takes place at a set of cluster heads) to fully distributed where computation takes place at every node.

\section{RSS Techniques}

A popular set of approaches tries to employ the received signal strength (RSS) as distance estimate between a wireless sender and a receiver. If the physical signal itself can not be measured, packet loss can be used to estimate RSS (Royer, 1999). But the relation between the RF signal strength and spatial distances is very complex as real world environments do not only consist of free space. They also include various objects that cause absorption, reflection, diffraction, and scattering of the RF signals (Rappaport, 1996). The transmitted signal therefore most often reaches the receiver by more than one path, resulting in a phenomenon known as multi path fading (Neskovic et. al, 2000). The quality of these techniques is hence very limited; the spatial resolution is restricted to about a meter and there are tremendous error rates (Xang et. al. 2005). They are hence mainly suited for Context-Awareness with room or block resolution (Yin et. al, 2005). Due to the principle problems with RSS, there are attempts to develop dedicated hardware for localizing objects over medium range. 


\subsection{Properties}

The indoor environment poses additional challenges compared to the outdoor environment. The channel is complex; having various multipath, scattering and diffraction effects that make estimating the signal strength highly probabilistic. Traditional methods working based on AoA and ToF principles can not be used with this technology, additionally special hardware is required to get a time synchronized network out of the available standard access points. Therefore the only applicable method is the received signal strength technique. RSS is the simplest RF measurement technique as its values are easily accessible through a WLAN interface. It is more preferred than the Signal to Noise ratio (SNR) to be used for the radio signature, because it is more location dependant (Bahl \& Padmanabhan, 2000). Noise can vary considerably from location to location and may heavily depend on the external factors, while this is not the case for the received signal strength. Since the RSS values still significantly fluctuate over time for a given location, they can be considered as a random variable, and hence, are described in a statistical fashion in order to estimate the distribution parameters. The fundamentals of the RSS techniques come from the Frii's Transmission Equation. The reason that Friis' formula does not work satisfactorily for indoor propagation is that communicating points may suffer from nonl Line of Sight condition (nLoS). Strength decay of the received signal not only comes from path loss, but also shadowing and fading distort the received signal quality. They both depend on the environmental features, barriers and occlusion. Short scale fading due to multipath, adds random high frequency terms with large amplitude (Rappaport, 1996). This issue is more effective indoors. Still because of less complexity that the hardware design and implementation phases need, the RSS solution has been in the field of interest amongst most of the localization researches.

\subsection{Techniques}

Apart from the statistical or probabilistic representation of signals, there are essentially two categories of RSS based techniques for positioning using WLAN: Trilateration and Location Fingerprinting. The prerequisite of the trilateration method is using a signal propagation model to convert RSS measurement to a transmitter-receiver (T-R) separate distance estimate. Utilizing the general empirical model can only obtain a very inaccurate distance of $\mathrm{T}-\mathrm{R}$, therefore a more accurate and correction-enforced model is required. Before a positioning system can estimate the location, a fingerprint database (also referred to as a radio map) constructed. In other words, a "Location Fingerprinting" localization system consists of two phases, the offline (training) and the online (localization) phase. During the online phase a site survey is performed by measuring the RSS values from multiple APs. The floor is divided into predefined grid points. The RSS values are measured at desired locations on the grid. Multiple measurements are taken and the average values are stored in a database. The location fingerprint refers to the vector of RSS values from each access point and their corresponding location on the grid. A reference carpet refers to the collection of fingerprints and their associated locations for a given area. The drawback here is the extensive training values that have to be predetermined, separately for each particular indoor environment.

Understanding the statistical properties of the location fingerprint (aka. RSS vector) is important for the design of a positioning system due to several reasons. It can provide insights into how many APs are needed to uniquely identify a location (Kaemarungsi \& 
Krishnamurthy, 2004) with a given accuracy and precision, and, whether preprocessing of the RSS measurements can improve such accuracy. Within the same perspective, (Li et. al, 2005) has proposed a hybrid method compose of two stages.

Area localization has been seen as a more viable alternative compared to point based localization mainly due to the fact that they can better describe localization uncertainty (Elnahraway et. al, 2004). They can easily trade accuracy for precision. Accuracy is the likelihood of the object being within the returned area, while precision is the size of the returned area. Point based algorithms on the other hand have difficulty in describing this behaviour. It was found that although area based approaches better described the localization uncertainty, their absolute performance is similar to point based approaches (Elnahraway et. al, 2004).

\section{Physical and Logical Topology of WSN}

Field of networked robotics is envisioned to be one of the key research areas in robotics recently (Bekey et. al, -). This research field, flourishing in the last decade or so, is gaining additional momentum thanks to the advent of cheap, easy to deploy and low power nodes to build sensor networks. Richness of different sensors, and their prompt availability open new scenarios to solve some long standing problems.

In this scenario, plenty of wireless sensors are deployed to the environment, in order to build up a network. This network first of all aims for holding a full connectivity which in most of applications can be represented by a graph. Plenty of theories and properties of graphs can merge to the scenario and open new doors for improvements (Gotsman \& Koren, 2005). However full network connectivity has its own advantages, local connectivities in order to extract connected clusters also are shown to be sufficient under some circumstances (Chan et. al, 2005).

Assume a scenario that builds a mobile wireless (-sensor) network with possibly physical dynamic topology. If there are enough relative distances information available, it is not quite complicated to use some multilateration technique for finding a position, but it is conditioned on the availability of enough anchors to be used as references (Khan et. al, 2006). In this class, many solutions assume the availability of detectable landmarks at known positions in order to implement, for example, Kalman based localization methods (Leonard \& Durrant, 1991) and (Pillonetto \& Carpin, 2007), while some other approaches are developed based on anchor-free combinations (Pryantha et. al, 2003). Here, we explicitly solve the following layout problem: Given a set of mobile robots (simply named nodes) which are equipped with wireless transceivers and a mechanism by which each node can estimate its distance to a few nearby ones (or even through the whole network), the goal is to determine coordinates of every node via local or global communication.

In general, radio communication constraints are a set of geometry rules to bound position estimates. Such constraints are a combination of radial and angular limitations. Latter aspects are out of the interests of this research. Ranging information is adequately sufficient in for these achievements. It goes to the fact that if all internode wireless links are established and their associative lengths are known, their graph representation is indeed uniquely realized. When only one subset of distances is known, more sophisticated techniques must be used. In contrast, when multiple solutions exist, the main phenomenon observed is that of foldovers, where entire pieces of the graph fold over on top of others, 
without violating any of the partial distance constraints. A main challenge is to generate a solution which is fold-free. Occasionally, final result may suffer from translation, rotation and reflection degrees of freedom, but either of these are not important, because can be resolved by assigning some known coordinates to any three arbitrary nodes.

Problem of reconstructing a geometric graph given its edges' lengths, has received some attention in discrete as well as computational geometry communities, whereas it is also relevant for molecule construction and protein folding applications, psychology, networking and also mobile robotics. Our main aim here is to roughly show how to extend graph realization analogy with noisy edges, for localizing the nodes with higher likelihood of unambiguous realization.

In an interconnected network of $n$ nodes, if all distances are known exactly, it is only possible to determine the relative coordinates of the points, i.e., to calculate the point coordinates in some arbitrary system. To place the points in an absolute coordinate system, it is necessary to know the positions of at least $D+1$ points, where $D$ indicates the dimension of the space. Consider that a set of $k$ anchor points are having a known position in the 2D space. A set of $n-k$ nodes with unknown positions can possibly be spatially localized based on the known positions of those anchors. If $k=0$, the positioning scenario is called relative or anchor-less localization.

On the other hand, an incremental approach can deal with a large size network where nodes are having a limited range of communication and indeed ranging. This solution, assigns coordinates to a small core of nodes, and repeatedly, updates coordinates to more neighbors based on local calculations and then proceeds until the whole network is covered. This solution is totally error prone in the early stages, unless being reinforced by proper filtering methods and statistical approaches, is very likely to break the error upper supremum.

\section{Conclusions}

In this chapter, after surveying traditional methods for robot localization, we more emphasized on the radio networks and their ranging capabilities including RSS in WLAN networks as well as ToF measurements of WSN topologies. Their positioning accuracy is comparable in practice where the latter provides quite better positioning results. Practical results indicate that the last approach, enforced with some more mathematical representation can be reliably used for variety of mobile robotics positioning applications both indoor and outdoors, relatively independent from size of the mobile robotics network, explicitly explained in (Bastani \& Birk, 2009). In other words, having ranging capabilities and specially by radio signals enables the robots to overcome localization problems, less dependently from error accumulation and environmental features.

\section{References}

Thrun, S (2002). Robotic mapping: A survey, Exploring Artificial Intelligence in the New Millennium, Morgan Kaufmann.

Lee, B; Cai, W,; Turner, J. \& Chen, L. (?). Adaptive Dead Reckoning Algorithms for distributed Interactive Simulation, Journal of SIMULATION, Vol. 1, No 1-2.

Heller, A. K. \& Taylor, S. (?). Using Determinism to Improve the Accuracy of Dead Reckoning Algorithms. Australian National University, CANBERRA. 
Roos, T. (2002). A probabilistic approach to wlan user location. Intl Journal of WirelessInformation Networks, Vol 9.

Birk, A. (1996). Learning geometric concepts with an evolutionary algorithm. Procedings of The Fifth Annual Conference on Evolutionary Programming. The MIT Press, Cambridge.

Salazar, A.E.S. (2004). Positioning bluetooth and Wi-Fi systems, IEEE Trans. Consumer Electron., Vol. 50, No. 1, pp. 151-157.

Krishnan, P., Krishnakumar, A.S., Ju, W.H., Mallows, C. \& Ganu, S. (2004) A system for LEASE: System for location estimation assisted by stationary emitters for indoor RF wireless networks, Proceedings of IEEE Infocom, pp. 1001-1011. Hong Kong.

Ganu, ., Krishnakumar, A.S. \& Krishnan, P. (2004). Infrastructure-based location estimation in WLAN, Proceedings of IEEE Wireless Communications and Networking Conf. (WCNC 2004), pp. 465-470.

Kritzler, M.; Lewejohann, L.; Krger, A.; Raubal, M. \& Sachser, N. (2006). An RFID-based Tracking System for Laboratory Mice in a Semi-Natural Environment, Proceedings In PTA2006 Workshop, Pervasive Technology Applied Real-World Experiences with RFID and Sensor Networks.

Fontanaand, R. \& Gunderson, S. (2002). Ultra wideband precision asset location system, Proc. IEEE Conf. Ultra Wideband Systems and Technologies, pp. 147-150.

Lee, J.Y. (2002). Ultra-wideband ranging in dense multipath environments, Ph.D. dissertation, Dept. Elect. Eng., Univ. Southern California.

Royer, E.M. \& Toh, C.K. (1999). A review of current routing protocols for ad-hoc mobile wireless networks. IEEE Personal Communications Magazine, pp. 46-55.

Rappaport, T.S. (1996). Wireless Comm. Principles and Practice. IEEE Press /Prentice Hall.

Neskovic, A.; Nescovic, N. \& Paunovic, G. (2000). Modern approaches in modelling of mobile radio systems propagation environment. IEEE Communications Surveys.

Xiang, Z.; Zhang, H.; Huang, J.; Song, S.\& Almeroth, K. (2005). A hidden environment model for constructing indoor radio maps. Sixth IEEE International Symposium on a World of Wireless Mobile and Multimedia Networks (WoWMoM 2005). pp. 395-400.

Yin, J.; Yang, Q.\& Ni, L. (2005). Adaptive temporal radio maps for indoor location estimation. 3rd IEEE international conf. on Pervasive Computing and Communications, (PerCom 2005). pp. 85-94.

Bahl, P \& Padmanabhan, V.N. (2000). RADAR: An in-building, RF based user location and tracking system, Proceedings of IEEE Infocom 2000, Vol. 2, pp. 775-784. Tel-Aviv, Israel.

Rappaport, T. (1996). Wireless Communications Principle and Practice, Prentice Hall.

Kaemarungsi, K. \& Krishnamurthy, P. (2004). Modeling of Indoor Positioning Systems Based on Location Fingerprinting. Proceedings of IEEE INFOCOM 2004.

Li, B.; Dempster, A.; Rizos, C. \& Barnes, J. (2005). Hybrid Method for Localization Using WLAN. School of Surveying and Spatial Information System, University of New South Wales, Sydney.

Elnahraway, E.; Li, X. \& Martin, R. P., (2004). The limits of localization using RSS. Proceedings of the 2nd international conference on Embedded networked sensor systems, pp. 283-284, New York, USA.

Sklar. B.(1997). Rayleigh fading channels in mobile digital communication systems, IEEE Communications Magazine, Vol. 35, pp. 90-100.

Thrun, S. (2000). An Online Mapping Algorithm for Teams of Mobile Robots, CMU-CS-00-167. 
Bekey, G.; Ambrose, R.;V. Kumar ; A. Sanderson ; B. Wilcox, \& Y. Zheng. (?). International assessment of research and development in robotics.

Gotsman C. \& Koren, Y. (2005). Distributed graph layout for sensor networks, Journal of graph algorithms and applications, Vol.9, No.1.

Chan, H.; Luk M. \& Perrig, A. (2005). Using Clustering Information for Sensor Network Localization, LNCS, Springer, Distributed Computing in Sensor Systems, Vol. 3560, pp. 109-125.

Khan, H.M. ; Olariu, S. \& Eltoweissy, M., (2006). Efficient single-anchor localization in sensor networks, Dependability and Security in Sensor Networks and Systems, DSSNS.

Bastani, H. \& Birk, A. (2009). Precise Realtime Localization by RF Transceiver ToF Measurements, 14th International Conference on Advanced Robotics (ICAR), Germany.

Leonard, J. \& Durrant-Whyte, H. (1991). Mobile robot localization by tracking geometric beacons, IEEE Transaction on Robotics and Automation, Vol.7, No. 3, pp.:376-382.

Pillonetto, G. \& Carpin, S. (2007). Multirobot localization with unknown variance parameters using iterated Kalman filtering, IEEE/RSJ International Conference on Intelligent Robots and Systems. pp.: 1709-1714.

Priyantha, N.; Balakrishnan, H.; Demanie, E. \& Teller, S. (2003). Anchor-free distributed localization in sensor networks, Proceedings of SenSys 03.

Horn, K. (1987). Closed-form solution of absolute orientation using unit quaternion, Journal of Optical Society. USA, Vol.4, pp.: 629-638.

Wang, J.; Chi, V. \& Fuchs, C. (1990). A Real-time Optical 3D Tracker for Head-mounted Display Systems, Computer Graphics, ACM SIGGRAPH, Vol. 24, No.2, pp.205-215.

Choi, Y. \& Oh, S. (2005). Visual Sonar Based Localization Using Particle Attraction and Scattering, Proceedings of the IEEE International Conference on Mechatronics and Automation, Canada.

Carpin, S. \& Birk, A. (2004). Stochastic map merging in rescue environments, In Robocup 2004. Springer.

Fox, D.; Thrun, S.; Burgard, W. \& Dellaert, F. (2001). Particle filters for mobile robot localization. Sequential Monte Carlo Methods in Practice, Springer Verlag, pp. 499-516.

Bastani, H. \& Mirmohammad Sadeghi, H.; Shariatmadari, A.; Azarnasab, E. \& Davarpanah Jazi, M. (2005). Design and Implementation of a Full Autonomous Mine Detecting Robot, 5th IFAC on Intelligent Autonomous Vehicles - IAV04, Elsevier, ISBN 008 0442374.

Pfingsthorn, M. \& Birk, A. (2008). Efficiently Communicating Map Updates with the Pose Graph, International Conference on Intelligent Robots and Systems (IROS), 2008.

Qing, H. (2005) A compact ESPAR antenna with planer parasitic elements on a dielectric cylinder, IEICE trans.communication. Vol.E88-B.

Shimizu, M. (2005). Tiny Seekers, Robocup Rescue - Robot League Team, TDP in Robocup 2005 proceeding (CD format), Japan. 


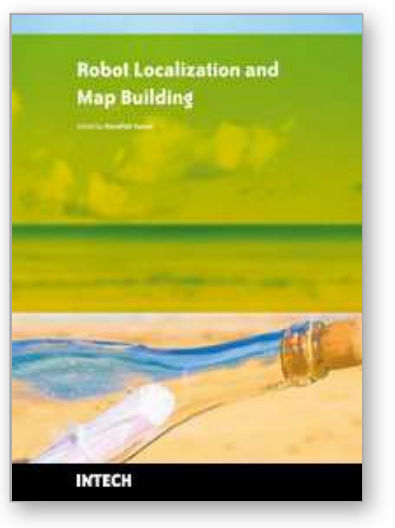

\author{
Robot Localization and Map Building \\ Edited by Hanafiah Yussof
}

ISBN 978-953-7619-83-1

Hard cover, 578 pages

Publisher InTech

Published online 01, March, 2010

Published in print edition March, 2010

Localization and mapping are the essence of successful navigation in mobile platform technology. Localization is a fundamental task in order to achieve high levels of autonomy in robot navigation and robustness in vehicle positioning. Robot localization and mapping is commonly related to cartography, combining science, technique and computation to build a trajectory map that reality can be modelled in ways that communicate spatial information effectively. This book describes comprehensive introduction, theories and applications related to localization, positioning and map building in mobile robot and autonomous vehicle platforms. It is organized in twenty seven chapters. Each chapter is rich with different degrees of details and approaches, supported by unique and actual resources that make it possible for readers to explore and learn the up to date knowledge in robot navigation technology. Understanding the theory and principles described in this book requires a multidisciplinary background of robotics, nonlinear system, sensor network, network engineering, computer science, physics, etc.

\title{
How to reference
}

In order to correctly reference this scholarly work, feel free to copy and paste the following:

Hamed Bastani and Hamid Mirmohammad-Sadeghi (2010). Ranging Fusion for Accurating State of the Art Robot Localization, Robot Localization and Map Building, Hanafiah Yussof (Ed.), ISBN: 978-953-7619-83-1, InTech, Available from: http://www.intechopen.com/books/robot-localization-and-map-building/ranging-fusionfor-accurating-state-of-the-art-robot-localization

\section{INTECH}

open science | open minds

\section{InTech Europe}

University Campus STeP Ri

Slavka Krautzeka 83/A

51000 Rijeka, Croatia

Phone: +385 (51) 770447

Fax: +385 (51) 686166

www.intechopen.com

\section{InTech China}

Unit 405, Office Block, Hotel Equatorial Shanghai

No.65, Yan An Road (West), Shanghai, 200040, China

中国上海市延安西路65号上海国际贵都大饭店办公楼 405 单元

Phone: +86-21-62489820

Fax: $+86-21-62489821$ 
(C) 2010 The Author(s). Licensee IntechOpen. This chapter is distributed under the terms of the Creative Commons Attribution-NonCommercialShareAlike-3.0 License, which permits use, distribution and reproduction for non-commercial purposes, provided the original is properly cited and derivative works building on this content are distributed under the same license. 